\title{
Fermented Brown Rice and Rice Bran with Aspergillus Oryzae as A New Promising Agent for Cancer Prevention
}

\author{
Hideki Mori* \\ Department of Tumor Pathology, Gifu University Graduate School of Medicine, Japan \\ *Corresponding author: Hideki Mori, Department of Tumor Pathology, Gifu University Graduate School of Medicine, Japan. \\ To Cite This Article: Hideki Mori, Fermented Brown Rice and Rice Bran with Aspergillus Oryzae as A New Promising Agent for Cancer Prevention. \\ Am J Biomed Sci \& Res. 2019 - 6(3). AJBSR.MS.ID.001035. DOI: 10.34297/AJBSR.2019.06.001035.
}

Received: 眥 November 19, 2019; Published: 眥 November 25, 2019

\section{Minireview}

Fermented brown rice and rice bran with Aspergillus (A) oryzae (FBRA) is a food manufactured by fermenting a mixture of brown rice and rice bran with $A$. oryzae. The fermentation is proved to increase antioxidant phytochemicals. FBRA prevented carcinogeninduced carcinogenesis in 7 animal models. FBRA also acted against spontaneous development of neoplasms in 3 animal models. FBRA is suggested to be a new promising agent for prevention of human cancers. It has been recognized that dietary factors play an important role in prevention of occurrence of human cancers. Epidemiological studies have shown that high intake of fruits, vegetables and cereal foods decrease the risk for the occurrence of cancers in different organs. Rice is one of the major cereals, and is the most consumed food worldwide, particularly in Asian countries. Rice seeds and rice bran contain fibers and various types of antioxidants including phenolic acids, phytic acid and tocopherols. Cellular and preclinical studies have supported that micronutrients in rice bran have effects against the occurrence of several types of cancers. Our group has done a large-scale study to determine possible agents to suppress occurring cancers using animal models and found a number of natural or synthetic substances including rice-germ [1,2].

FBRA is a food manufactured by fermenting a mixture of brown rice and rice bran with $A$. oryzae to improve its digestibility. Basic composition of FBRA and its manufacturing process are shown previously $[3,4]$. It is already known that FBRA has fermentationinduced increase of antioxidant phytochemicals [5,6] and acts as a potent free radical scavenger [7]. Among the antioxidant phytochemicals contained in FBRA, polyamines, especially spermidine is known to play a central role in cell proliferation and growth [6]. Recent another study revealed that FBRA possesses a property to induce apoptosis in human colorectal tumor cells [8] and inhibits inflammatory-related carcinogenesis in mice [9]. Our group carried out experimental studies to confirm possible preventive effects on cancer development using different animal models. FBRA was supplied by Genmai Koso Co., Ltd, (Sapporo,Japan). Indeed, FBRA prevented carcinogen-induced tumorigenesis in a number of organs such as colon (azoxymethane-induced rat model) [3], pancreas (N-nitrosobis(2-oxopropyl)amine-induced hamster model) [4], liver (diethylnitrosoamine and phenobarbital-induced rat model) [10], esophagus (N-nitrosomethylbenzylamine-induced rat model) [11], stomach (N-methyl-N'-nitro-N-nitrosoguanidineinduced rat model) [12], urinary bladder (N-butyl-N-(4hydroxybutyl)-nitrosoamine-induced mouse model) [13] and lung (4-(methylnitrosoamino)-1-(3-pyridyl)-1-butanone-induced mouse model) [14].

Thus, FBRA prevented carcinogen-induced carcinogenesis in almost all representative rodent models except mammary carcinogenesis in rats with use of 7,12-dimethylbenz[a]anthracene [unpublished results]. Interestingly, FBRA suppressed spontaneous carcinogenesis of prostate in a transgenic TRAP rat (in this rat, hormonally regulated rat probasin promotor specifically drives the expression of SV40 $\mathrm{T}$ antigen in the prostatic epithelium) [15] and thymic lymphomatogenesis in a mouse model (AKR/NSlc female mice)[16]. Although FBRA did not exhibit preventive effect on the spontaneous development of colon tumors in ApcMin/+ mice, the agent clearly indicated prevention of carcinogenesis in dextran sodium sulfate-exposed ApcMin/+ mice [17]. In the studies using 
chemical carcinogens, exposure of FBRA decreased multiplicity as well as incidence of neoplasms including carcinomas. In principle, effect of FBRA was apparent by exposure during the post-initiation phase (term after the administration of carcinogen) [3,4,10-14].

FBRA did not have clear preventing effect by exposure during the initiation phase (term during the administration of carcinogen) suggesting that effect of FBRA is not related to metabolic activation or detoxication of carcinogen. Furthermore, FBRA decreased carcinogen-induced cell proliferation in non-neoplastic area of organs for the carcinogenesis $[3,10,11,13]$. Present author already stated that control of cell proliferation is quite important for the underlying mechanism of cancer chemoprevention [18]. For the case of FBRA against prostate carcinogenesis in TRAP rats, retardation of transition of preneoplastic lesions to neoplasms seemed to be important [15]. As mode of action of FBRA for the inhibition of thymic lymphomatogenesis in mice, emergence of cellular apoptosis being regarded as a mechanism of cancer prevention, in the preneoplastic and neoplastic cells was suggested to be a key issue [16]. Through the all animal experiments for modifying effects of FBRA, FBRA itself did not exert any toxic changes in any organs histologically. Accordingly, FBRA is suggested to be a prominent safety agent for prevention of human cancers, and our data emphasize the advantage of unpolished rice as a daily staple food.

\section{References}

1. Mori H, Tanaka T, Sugie S, Yoshimi N, Kawamori T, et al. (1997) Chemoprevntion by naturally occurring and synthetic agents in oral, liver and large bowel carcinogenesis. J Cell Biochem Suppl 27: 35-41.

2. Kawabata K, Tanaka T, Murakami T, Okada T, Murai H, et al. (1999) Dietary prevention of azoxymethane-induced carcinogenesis with ricegerm in F 344 rats. Carcinogenesis 20(11): 35-41.

3. Katayama M, Yoshimi N, Yamada Y, Sakata K, Kuno T, et al. (2002) Preventive effect of fermented brown rice and rice bran against colon carcinogenesis in male F344 rats. Oncol Rep 9(4): 817-822.

4. Kuno T, Takahashi S, Tomita H, Hisamatsu K, Hara A, et al. (2015) Preventive effects of fermented brown rice and rice bran against $\mathrm{N}$-nitrosobis (2-oxopropyl) amine-induced pancreatic tumorigenesis in male hamsters. Oncol Lett 10(6): 3377-3384.

5. Ogawa S, Takafuji K, Tsubuku S, Horie Y, Ikegawa S, et al. (2017) Isotope-coded derivatization based LC/ESI-MS/MS methods using a pair of novel reagents for quantification of hydroxycinnamic acids and hydroxybenzoic acids in fermented brown rice product. J Pharmace.
Biomed Analysis 142: 167-170.

6. Horie Y, Goto A, Tsubuku S, Itoh M, Ikegawa S, et al. (2019) Changes in polyamine content in rice bran due to fermentation with Aspergillus oryzae analyzed by LC/ESI-MS/MS combined with derivatization. Anal Sci 35(4): 427-432.

7. Tazawa K, Oita N, Namikawa H (1999) Superoxide scavenging effect of fermented brown rice determined by ESR spin-trapping method. Food Style 21 (in Japanese). 3: 32-37.

8. Itoh M, Nishibori N, Sagara T, Horie Y, Motojima A, et al. (2012) Extract of fermented brown rice induces apoptosis of human colorectal tumor cells by activating mitochondrial pathway. Phytother Res 26(11): 16611666.

9. Onuma K, Kanda Y, Ikeda S, Sakaki RN, Nonomura T, et al. (2015) Fermented brown rice and rice bran with Aspergillus oryzae (FBRA) prevents inflammation-related carcinogenesis in mice, through inhibition of inflammatory cell infiltration. Nutrients 7: 10237-10250.

10. Katayama M, Sugie S, Yoshimi N, Yamada Y, Sakata K, et al. (2003) Preventive effect of fermented brown rice and rice bran on diethylnitrosoamine and phenobaribital-induced hepatocarcinogensis in male F344 rats. Oncol Rep 10: 875-880.

11. Kuno T, Hirose Y, Hata K, Kato K, Qiang Z, et al. (2004) Preventive effect of brown rice and rice bran on $\mathrm{N}$-nitrosomethylbenzylamine-induced esophageal tumorigenesis in rats. Int J Oncol 25(6): 1809-1815.

12. Tomita H, Kuno T, Yamada Y, Oyama T, Asano N, et al. (2008) Preventive effect of fermented brown and rice bran on N-methyl-N'-nitro-Nnitrosoguanidine-induced gastric carncinogenesis in rats. Oncology Reports 19(1): 11-15.

13. Kuno T, Hirose Y, Yamada Y, Hata K, Qiang Z, et al. (2006) Chemoprevention of mouse urinary bladder carcinogenesis by fermented brown rice and rice bran. Oncol Rep 15: 533-538.

14. Phutthaphadoog S, Yamada Y, Hirata A, Tomita H, Taguchi A, et al. (2009) Chemopreventive effects of fermented brown rice and rice bran against 4-(methylnitrosoamino)-1-(3-pyridyl)-1-butanon-induced lung tumorigenesis in female A/J mice. Oncol Rep 21: 321-327.

15. Kuno T, Nagano A, Mori Y, Kato H, Nagayasu Y, et al. (2016) Preventive effects of fermented brown rice and rice bran against prostate carcinogenesis in TRAP rats. Nutrients 8(7): E421-432.

16. Kuno T, Nagano A, Mori Y, Nagayasu Y, Tanaka T, et al. (2018) Preventive effects of fermented brown rice and rice bran on spontaneous lymphomatogenesis in AKR/NSlc female mice. Asian Pac J Cancer Prev 19: 3217-3233

17. Phuttgaphadoong S, Yamada Y, Hirata A, Tomita H, Hara A, et al. (2010) Chemopreventive effect of fermented brown rice and rice bran (FBRA) on the inflammation-related colororectal carncinogenesis in APCMIN/+ mice. Oncol Rep 23: 53-59.

18. Mori H, Sugie S, Yoshimi N, Hara A, Tanaka T, et al. (1999) Control of cell proliferation in cancer prevention. Mutat Res 428(1-2): 291-298. 